\title{
Una nueva gobernanza en el Fonadin como estrategia para eficientar las inversiones en infraestructura
}

Daniel César Ordóñez Bustos

Universidad Anáhuac México

Artículo recibido el 28 de enero y aprobado el 24 de abril de 2019

\section{Resumen}

Contar con la infraestructura necesaria en cuanto a cantidad y calidad, le permite a cualquier país instrumentar programas con alto impacto económico y social. En México hay un déficit importante en la materia, razón por la cual ocupa el lugar 62 en el índice de competitividad del World Economic Forum, debido entre otros factores a la disminución de la inversión como porcentaje del producto interno bruto (PIB) desde 2010. De ahi la demanda de recursos, en donde el Fondo Nacional de Infraestructura (Fonadin) tiene un papel relevante como instrumento del gobierno federal en materia de inversión pública. Sin embargo, el $67 \%$ de sus recursos se han canalizado a apoyos no recuperables y, por ello, requiere una nueva gobernanza, de modo que tome decisiones sobre criterios de eficiencia y viabilidad financiera.

Palabras clave: inversión pública; Fonadin y gobernanza.

Clasificación JEL: H54. 


\section{Abstract}

For any country, having the infrastructure in terms of resources and the required expertise in execution allows it to implement programs with high economic and social impact. In Mexico there is a significant deficit in this area so it ranks number 62 in the infrastructure competitiveness index of the World Economic Forum due, among other factors, to the decrease in investment as a percentage of gross domestic product $(G D P)$ since 2010. So the demand of resources where the National Infrastructure Fund (Fonadin) has a relevant role as a federal government public investment agency. However, $65 \%$ of its resources have been assigned to non-recoverable support and, therefore, a new governance is required to make decisions on criteria of efficiency and financial viability.

Keywords: public investment, Fonadin and governance.

JEL Classification: H54. 


\section{Introducción}

Los proyectos de infraestructura, ya sea que se trate de un sistema de movilidad urbana, una carretera, un hospital o una planta de generación eléctrica, tienen el potencial de transformar la dinámica económica y social de un país, ya que las inversiones en estos rubros revisten una doble importancia: por una parte, los recursos invertidos en obras y redes públicas presentan un efecto multiplicador en cuanto a empleo, ingreso y consumo. Por otra, una vez que se ponen en operación las redes públicas mencionadas, se ofrecen bienes y servicios que generan los medios e incentivos para fortalecer y ampliar la integración regional en un territorio nacional.

Precisamente por esos múltiples impactos, los gobiernos buscan los medios y alternativas para obtener los recursos demandados para este tipo de iniciativas. Asimismo, resulta interesante analizar el desarrollo de la infraestructura en el país en los últimos años, así como los agentes que han participado en su financiamiento. En este contexto, se propone considerar el papel del Fondo Nacional de Infraestructura (Fonadin) como una de las principales instancias del gobierno federal para financiar proyectos de alto impacto social. Del mismo modo, y derivado del análisis, se plantea un esquema de decisiones que aporte elementos y criterios en el momento de decidir qué proyectos deben financiarse con los recursos públicos que maneja el Fondo.

Alineado con este objetivo, el documento se divide cinco secciones. Después de esta introducción, la segunda sección se enfoca en los conceptos teóricos que servirán de soporte, tanto para el análisis, como para la propuesta de esquema de decisiones, ubicados en el ámbito de la microeconomía. En el tercer apartado se revisan los mecanismos a través de los cuales las inversiones impactan en el crecimiento y desarrollo económico, incluyendo una referencia a investigaciones y estudios empíricos sobre estas relaciones. La sección cuarta integra un diagnóstico de la infraestructura nacional, identifica el papel del Fonadin y describe su participación en el mercado de financiamiento de proyectos.

A partir del diagnóstico y con base en las Reglas de Operación del Fonadin (ROP), se formula un esquema de cambio en su gobernanza, con la finalidad de que las decisiones en la selección de proyectos estén sustentadas en criterios de eficiencia y rentabilidad económica y financiera, para al final incluir las conclusiones generales del análisis. 


\section{Marco teórico}

Como sustento para el análisis y los alcances propuestos en este artículo, se sugiere recurrir a los conceptos básicos de la microeconomía. Es importante recordar que esta rama de la economía se enfoca en el estudio de las elecciones de los agentes económicos en el uso de los recursos y de los costos de oportunidad incurridos. En el caso del gobierno, considera las decisiones sobre la asignación de recursos públicos entre distintas opciones, una de las cuales es la inversión en infraestructura.

Otro componente a tomar en cuenta corresponde a las características y supuestos del modelo de equilibrio en el mercado, así como las condiciones de eficiencia en la competencia perfecta, para lo cual cobra relevancia la estructura de costos económicos de las empresas.

Asimismo, como apoyo para la integración de la propuesta de cambio de gobernanza, se toman como referencia elementos de la teoría de la elección pública y los modelos de comportamiento económico de la burocracia, específicamente el desarrollado por Hartley y Tisdell (1981) con base en las aportaciones de William Niskanen (1971), cuyo objetivo es analizar el comportamiento de la burocracia en su búsqueda de maximizar utilidad y su relación con el presupuesto público.

A manera de resumen, en la siguiente tabla se presentan los conceptos que permiten explicar el comportamiento de cada uno de los agentes económicos involucrados en los proyectos de infraestructura, y que son la base para el desarrollo de los apartados de este documento:

Tabla 1. Agentes económicos y conceptos relacionados

\begin{tabular}{|l|l|l|}
\hline $\begin{array}{c}\text { Tomadores de } \\
\text { decisiones }\end{array}$ & \multicolumn{1}{|c|}{ Objetivos } & \multicolumn{1}{c|}{ Conceptos } \\
\hline Empresas & Utilidades & $\begin{array}{l}\text { Teoría de la firma } \\
\text { Teoría de la producción } \\
\text { Estructuras de mercado }\end{array}$ \\
\hline Gobierno & Beneficio social & $\begin{array}{l}\text { Economía del sector público } \\
\text { Economía del bienestar } \\
\text { Elección pública } \\
\text { Mecanismos de distribución }\end{array}$ \\
\hline
\end{tabular}

Fuente: elaboración propia. 
Sin lugar a dudas se ha convertido en un lugar común en distintas instancias afirmar que la infraestructura es básica para el funcionamiento eficiente de la economía y de la sociedad, toda vez que no es solo un motor de crecimiento sino también un factor de inclusión social. Lo anterior en virtud de que la construcción de puentes, carreteras, puertos y aeropuertos, así como el despliegue de redes de energía, agua y telecomunicaciones, articulan en el espacio geográfico el asentamiento de la población y generan las bases para la interacción de los agentes económicos y de los procesos productivos, de distribución y comercialización de bienes y servicios.

Debido a estas relaciones y repercusiones, la infraestructura se ha convertido en una esfera de interés académico. El análisis teórico acerca de la vinculación entre infraestructura, productividad y crecimiento tiene sus inicios en Arrow y Kurz (1970), quienes fueron los primeros en incluir el capital público como aporte a la función de producción agregada de la economía. Asimismo, la investigación empírica comenzó más tarde con Aschauer (1989), quien demostró que la inversión en ese campo tuvo un efecto significativo en el crecimiento económico de Estados Unidos. Años más adelante, Calderón y Servén (2002) demostraron que el estancamiento de la construcción de infraestructura en América Latina, durante los decenios de los ochenta y noventa, explica en parte el freno del crecimiento económico experimentado en ese período. En un estudio posterior, estos mismos autores señalan que el incremento conjunto de infraestructura creada entre los períodos quinquenales de 1991 a 1995 y de 2001 a 2005 aportó 1.1 puntos porcentuales de forma anual al crecimiento económico en la región.

Se tienen estudios más recientes como el de Standard \& Poor's (2015), en el cual se estima que el incremento en el gasto en infraestructura de $1 \%$ del PIB aumentaría el tamaño de la economía en $2.5 \%$ en Brasil, $1.4 \%$ en Canadá, $1.7 \%$ en Estados Unidos y $1.3 \%$ en México en un período de tres años.

Cabe añadir que el tema de la infraestructura ha sido también del interés de los organismos económicos internacionales. Por ejemplo, el FMI ha destacado que el dividendo económico de cerrar la brecha en la inversión pública puede ser sustancial en términos de eficiencia, ya que puede más que duplicar el impacto de tal inversión sobre el crecimiento. Su cuestionamiento se refiere a los instrumentos para financiarla: mediante deuda, impuestos o disminución de otras categorías del gasto, con lo que señalan que, si se cumplen determinadas condiciones, la inversión del gobierno puede ser eficiente, rentable y recuperarse totalmente. 
Por su parte, la Organización para la Cooperación y el Desarrollo Económicos (OCDE) ha publicado diversos documentos que resaltan factores como la calidad de la infraestructura y señalan que prácticamente todos los países se enfrentan a los mismos retos, reconociendo que la inversión pública es compleja toda vez que, desde su conceptualización hasta su construcción y explotación, está plagada de obstáculos y dificultades. Añaden que una de las principales razones por las que con frecuencia los proyectos de infraestructura no cumplen con el calendario y el presupuesto establecidos, ni con los objetivos previstos sobre el suministro de servicios, es una gobernanza deficiente.

Asimismo, el Foro Económico Mundial (WEF por sus siglas en inglés) publica anualmente el Índice Global de Competitividad, el cual evalúa los factores que impulsan la productividad y crecimiento en los países, y uno de sus indicadores es específicamente el que mide la calidad de la infraestructura. Este indicador incluye un análisis sobre las características de las redes carreteras, ferroviarias, portuarias, aeroportuarias, eléctrica y de telecomunicaciones.

En este contexto, como marco de referencia resulta importante analizar las características específicas de la infraestructura en el país.

\section{La infraestructura en México}

Con el cambio en la administración del gobierno federal, el tema de la infraestructura apareció en los primeros planos, tanto como factor de controversia en materia de política pública - con el caso del nuevo aeropuerto internacional, cuya ubicación se había previsto en Texcoco-, como instrumento de presión en la solución de problemas públicos, con el bloqueo de la Coordinadora Nacional de Trabajadores de la Educación del sistema ferroviario en Michoacán. En esa medida quedó de manifiesto la relevancia de este tema en la agenda económica, política y social del país, sustentando así la importancia de revisar su evolución.

En México, si bien se han realizado considerables esfuerzos en materia de inversión, persisten importantes brechas cuya consecuencia ha sido una provisión ineficiente de servicios y la ausencia de la infraestructura que demanda el crecimiento sustentable de la economía. Además, si se incluye en el análisis el criterio de calidad de la infraestructura, y no solo de su disponibilidad, el reto es aún más considerable.

A efecto de contextualizar estos elementos, se muestra la información del índice de competitividad en infraestructura del WEF, el cual incluye datos de 138 países sobre las redes principales de infraestructura: 


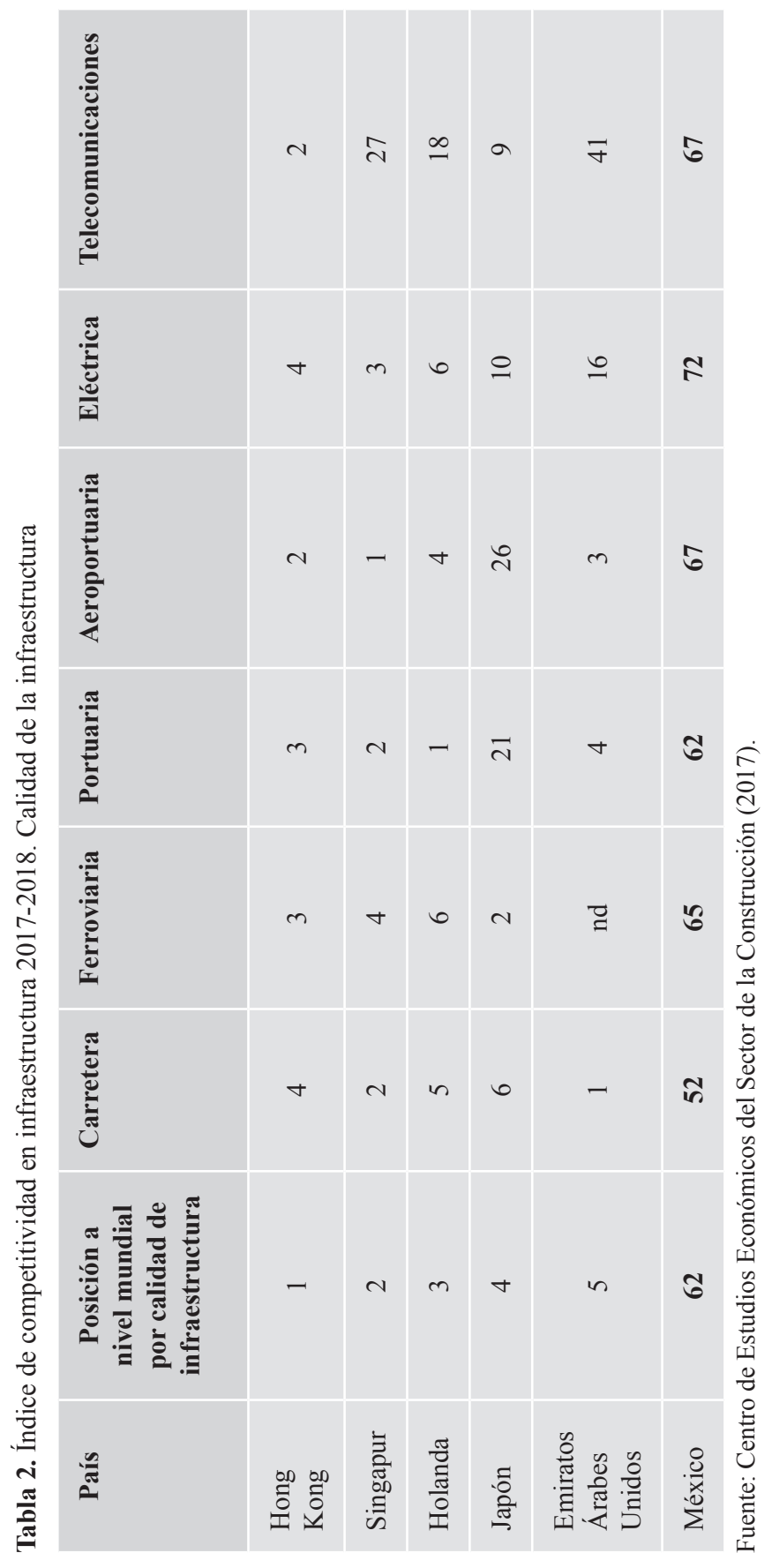


Como puede observarse, el país se ubica casi a la mitad del total de los países considerados (138), con una mejor infraestructura de carreteras y más deficiente en la cuestión eléctrica. Cabe señalar que México ha ido mejorando paulatinamente en estos rubros. De hecho, ha subido cuatro posiciones respecto al reporte de 2011-2012, pero estos avances no resultan significativos y las brechas que deben cerrarse aún persisten.

Por ello es importante analizar cuáles son los elementos que dan origen a estos resultados, con la finalidad de identificar posibles áreas de oportunidad que aporten a la solución de la problemática mencionada. En términos generales, los retos pueden agruparse en los siguientes dos rubros:

- Insuficientes flujos de inversión pública;

- La presencia de fallas institucionales, tanto en la conducción de las políticas públicas, como en la organización de los mercados.

\section{Inversión pública}

La primera variable de análisis es la evolución del monto de los recursos destinados a la inversión pública, ya que este rubro es el principal detonador del crecimiento de la infraestructura. A este respecto se cuenta con los siguientes datos correspondientes a los tres últimos sexenios:

Gráfica 1. Inversión pública como porcentaje del PIB en México

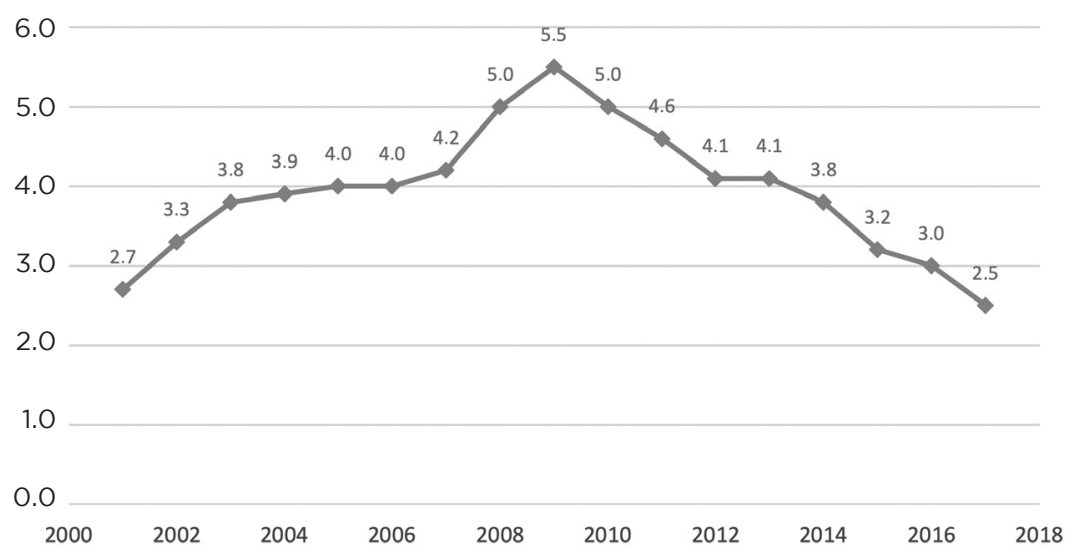

Fuente: Centro de Estudios Económicos del Sector de la Construcción (2017). 
De acuerdo con esta información, durante la administración del presidente Vicente Fox, la inversión pública como porcentaje del PIB mostró una tendencia relativamente ascendente. Ese mismo comportamiento se tiene en la primera parte del gobierno del presidente Felipe Calderón, sin embargo, en los siguientes años empieza a descender y lo sigue haciendo de forma sistemática durante toda la gestión del presidente Enrique Peña Nieto, aunque en el tercer trimestre de 2018 la tendencia se invierte y se registra nuevamente una participación de $3 \%$ del PIB, como en 2016.

Un dato que es relevante añadir es el que señalan Andrade y Lugo (2018), en el sentido de que entre 2011 y 2015 el $40 \%$ de los recursos totales de inversión pública se destinaron a Pemex y el resto al desarrollo del resto de la infraestructura nacional.

Para ofrecer una referencia que nos permita cotejar estas cifras, se tiene la siguiente información sobre inversión pública en algunos países para 2013:

Tabla 3. Inversión pública como porcentaje del PIB (2013)

\begin{tabular}{|l|c|}
\hline \multicolumn{1}{|c|}{ País } & Inversión pública como \% del PIB \\
\hline Francia & 4.0 \\
\hline Unión Europea & 2.9 \\
\hline Italia & 2.4 \\
\hline Brasil & 1.1 \\
\hline Chile & 1.1 \\
\hline
\end{tabular}

Fuente: Standard \& Poor's (2015).

Como es posible constatar, la inversión en México en el año 2017 es similar a la que tenían en la Unión Europea y en Italia y superior a la de los países de Sudamérica cuatro años antes.

Ante la escasez de fondos públicos, el gobierno ha buscado alternativas para obtener los recursos requeridos para financiar el desarrollo de la infraestructura. Una de estas son las asociaciones público-privadas, que se refieren a proyectos que se realizan bajo esquemas, como concesiones, arrendamientos, proyectos de prestación de servicios y obra pública financiada, en los que el sector privado provee infraestructura en forma total o parcial.

Con la finalidad de brindar certeza jurídica a este tipo de esquemas de inversión, se publicó en enero de 2012 la Ley de Asociaciones PúblicoPrivadas (Ley APP), a través de la cual se busca fomentar la participación 
del sector privado, con lo que además de capital de riesgo, aportan conocimientos técnicos y su experiencia como desarrolladores privados para la provisión de servicios públicos. A este respecto, vale la pena señalar que, si bien se concreta la participación de ambos sectores, el gobierno es el encargado de establecer la regulación, ya que el desarrollo de infraestructura trae aparejada la presencia tanto de externalidades positivas (efecto de red), como negativas (principalmente ambientales y sociales) y, en esa medida, es necesaria la intervención gubernamental para corregir las fallas del mercado.

De acuerdo con la información disponible en la página de internet de la Secretaría de Hacienda y Crédito Público, bajo la Ley APP se han estructurado doce proyectos (cinco de Comunicaciones y Transportes, cuatro del IMSS y tres del ISSSTE) y se encuentran en etapa de preparación 18 proyectos más (SHCP, 2016). Aun con estos esquemas de participación, no alcanza a revertirse la tendencia mostrada anteriormente en la gráfica 1 y la escasez de recursos públicos ha sido definitivamente un elemento que ha incidido en forma directa en el déficit de infraestructura en el país.

En este contexto, es importante mencionar que en 2011, la CEPAL (Comisión Económica para América Latina) hizo una estimación de distintas dimensiones de la brecha de infraestructura, concluyendo que un gasto anual promedio del orden de $5.2 \%$ del PIB era necesario en la región para poder afrontar los flujos de inversión en infraestructura requeridos para satisfacer las necesidades de las empresas y los consumidores finales entre 2006 y 2020 (CEPAL, 2011).

A la fecha, la CEPAL ha revisado esas estimaciones, en las que se refiere que la inversión para satisfacer las necesidades de infraestructura es ahora de $6.2 \%$ del PIB (CEPAL, 2017). En este mismo sentido, cabe recordar lo que se había señalado anteriormente referente a que la calificadora Standard \& Poor's sugiere que incrementar en México el gasto en infraestructura en $1 \%$ del PIB aumentaría el tamaño de la economía en $1.3 \%$ en un período de tres años.

\section{Instituciones y mercado}

Los recursos para el financiamiento de los proyectos de infraestructura en el país provienen principalmente del sector privado, organismos internacionales y del presupuesto público. En el primer caso, la aportación de los inversionistas privados se materializa tanto al comprometer capital directamente en los proyectos, como al participar en transacciones de capital y de deuda en la Bolsa de Valores y en mercados privados.

Uno de los instrumentos financieros disponibles en el ámbito bursátil son los fondos de inversión, los cuales permiten a los inversionistas diversificar sus portafolios en términos de capital y deuda. Actualmente existen las 
siguientes opciones: Certificados Bursátiles (CEBURES), Fideicomisos de Inversión y Bienes Raíces (FIBRAS), Certificados de Capital de Desarrollo (CKDs), Fondos de Capital Privado, Certificados de Proyectos de Inversión (CERPI) y los Fideicomisos de Inversión en Energía e Infraestructura (FIBRA E). En estos casos los rendimientos otorgados no son producto del pago de principal ni de intereses predeterminados, sino del usufructo y beneficios de cada proyecto. Por lo general se enfocan en impulsar proyectos de infraestructura, inmobiliarios, minería y desarrollo de tecnología.

Es importante señalar que, específicamente en los proyectos de infraestructura, cada una de estas opciones se enfoca en etapas particulares del ciclo de inversión. Así, las FIBRAS (Fideicomisos de Inversión y Bienes Raíces) y la FIBRA E (Fideicomisos de Inversión en Energía e Infraestructura) se relacionan con activos en operación (brownfields), mientras que los CKD (Certificados de Capital de Desarrollo, por sus siglas en inglés) y CERPI (Certificados de Proyectos de Inversión) se enfocan principalmente en nuevos proyectos (greenfields).

Adicionalmente se tiene la alternativa del financiamiento privado vía la banca comercial, la cual cobra relevancia porque puede asumir los riesgos que involucran las operaciones complejas de infraestructura, así como hacer frente a las asimetrías de información, en particular en las primeras fases del diseño y de operación de los proyectos. A manera de ejemplo, se tiene la siguiente información sobre los sectores y algunos proyectos a los cuales se otorga financiamiento:

Tabla 4. Participación de la banca comercial por sectores

\begin{tabular}{|l|l|l|}
\hline \multicolumn{1}{|c|}{ Banco } & \multicolumn{1}{|c|}{ Sector } & \multicolumn{1}{c|}{ Proyectos } \\
\hline Del Bajío & Agua y carreteras & $\begin{array}{l}\text { Planta de Tratamiento Tuchtlán } \\
\text { y Paso Limón (Chiapas) }\end{array}$ \\
\hline Banorte & Vivienda & $\begin{array}{l}\text { Desarrollo Urbano Integral } \\
\text { Sustentable Valle San Pedro } \\
\text { (Baja California) }\end{array}$ \\
\hline Inbursa & Carreteras & $\begin{array}{l}\text { Carreteras: Cardel-Poza Rica } \\
\text { y Tuxpan-Tampico. }\end{array}$ \\
\hline Santander & Agua y carreteras & Acueducto II en Querétaro. \\
\hline
\end{tabular}

Fuente: elaboración propia con información de las instituciones bancarias.

Asimismo, en los últimos años, diversos organismos multilaterales como el Banco Interamericano de Desarrollo, la Corporación Financiera Internacional, el Fondo Multilateral de Inversiones y el Banco de Desarrollo de 
América del Norte, entre otros, han apoyado el crecimiento y la modernización de infraestructura en México.

En lo que respecta al gobierno federal, su aportación se da principalmente a través de la inversión pública, así como con los programas de la Banca de Desarrollo, en donde tiene un papel preponderante el Fondo Nacional de Infraestructura.

\section{Fonadin}

El Fonadin es administrado por Banobras y tiene su antecedente en el Fondo de Inversión en Infraestructura (FINFRA) y en el Fideicomiso de Apoyo al Rescate de Autopistas Concesionadas (FARAC). Por ello se quedó como concesionario de una red de autopistas de cuota, la cual es operada por Caminos y Puentes Federales de Ingreso y Servicios Conexos (CAPUFE) y cuyos ingresos son los recursos que se utilizan para el financiamiento de proyectos básicamente en los siguientes sectores: comunicaciones, transportes, hidráulico, medio ambiente, desarrollo urbano y turismo. La particularidad de tales proyectos es que deben cumplir con un doble cometido: contar con alta rentabilidad social y ser un incentivo para la participación de la inversión privada, por lo que se procura que tengan también una rentabilidad económica.

La operación del Fonadin está regulada por reglas de operación (RO) en las cuales se establece que, para hacer frente a la demanda de recursos, tiene diversos instrumentos que se agrupan básicamente en dos grandes rubros: apoyos recuperables y apoyos no recuperables. De acuerdo con la información disponible, la distribución de los fondos es prácticamente de dos a uno en favor de estos últimos, como se observa en la siguiente gráfica:

Gráfica 2. Distribución de apoyos del Fonadin (cifras a diciembre de 2018)

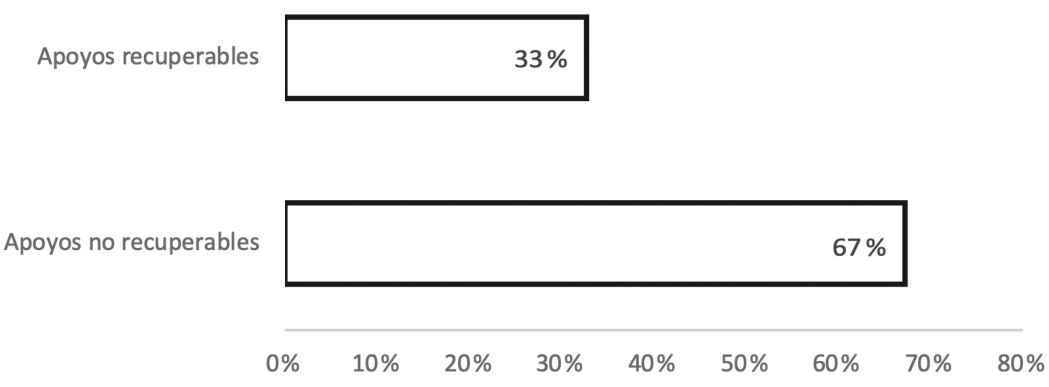

Fuente: Instituto Nacional de Transparencia, Acceso a la Información y Protección de Datos Personales, con datos del Banco Nacional de Obras y Servicios Públicos. 
En el caso de los apoyos recuperables, los recursos se destinan a proyectos que cuentan con una fuente de recuperación de manera que se aprovechan y nuevamente están disponibles y, con ello, los beneficiarios pueden acceder a opciones como las siguientes: créditos subordinados; líneas de crédito contingente; capital de riesgo, fondos de inversión y garantías.

Cabe señalar que prácticamente la mitad de los recursos de este rubro se destina a créditos subordinados y líneas de crédito contingente. Los primeros se refieren a la deuda dentro de la estructura de capital de un proyecto, la cual se paga después de cubrir cualquier obligación de deuda «senior» (emitida por un agente económico de la mejor calidad crediticia posible (Peiro, 2019) o preferente, así como las condiciones establecidas en la prelación de pagos. Adicionalmente, no tiene derecho a acceder ni a participar en las reservas adjudicadas para las obligaciones financieras senior, además de que los derechos de garantía sobre la liquidación otorgada a acreedores subordinados deberán clasificarse posteriormente a la deuda principal. ${ }^{1}$ Estos apoyos están concebidos para mejorar los flujos disponibles y la cobertura de la deuda bancaria o bursátil contratada para financiar proyectos.

Por su parte, la línea de crédito contingente es una alternativa que le permite a un estado o municipio cubrir faltantes del servicio o pago de la deuda de sus créditos contratados, así como las obligaciones de retribución a concesionarios que ofrecen los servicios en los proyectos financiados. Vale la pena señalar que, si bien los beneficiarios son precisamente estados y municipios, la prestación de los servicios es de las empresas concesionarias.

En este contexto, resulta interesante analizar a qué sectores se han destinado los apoyos recuperables, como lo muestra la gráfica 3 .

1 En las Reglas de operación del Fonadin se tiene la siguiente definición: «Créditos subordinados y/o convertibles.- Operaciones de financiamiento consistentes en deuda de carácter subordinado respecto de otros acreedores en proyectos de infraestructura, las cuales pueden ser convertidas a capital» (Fonadin, s/f). 
Gráfica 3. Distribución de apoyos recuperables (cifras a diciembre de 2018)

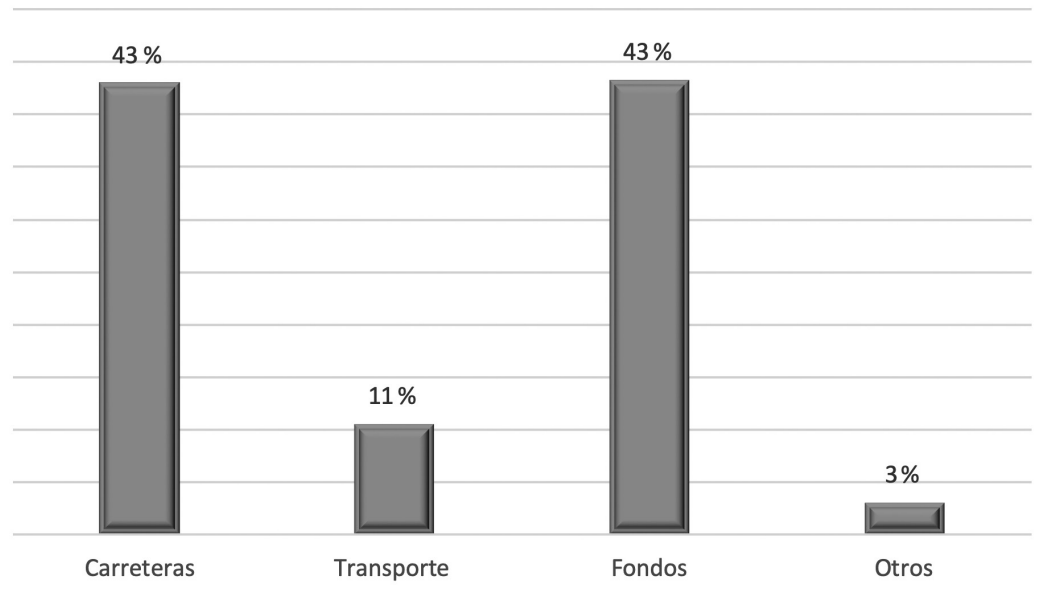

Fuente: Instituto Nacional de Transparencia, Acceso a la Información y Protección de Datos Personales, con datos del Banco Nacional de Obras y Servicios Públicos.

Como puede observarse en la gráfica $3, \$ 8$ de cada $\$ 10$ pesos de apoyo se destinaron a proyectos carreteros y a fondos de inversión. El Fonadin participa en estos instrumentos financieros desde $2010 \mathrm{y}$, a la fecha, lo hace en 19 fondos y dos fibras. De hecho, diez ya se encuentran en período de desinversión, con lo cual han reportado el reintegro de rendimientos sobre las inversiones. ${ }^{2}$ Con estos recursos se apoyan proyectos inmobiliarios, de energías limpias y renovables, turismo, carreteras, hidráulicos, hospitales, centros penitenciarios y plataformas logísticas, principalmente.

En lo que respecta a los apoyos no recuperables, son recursos proporcionados a dependencias y entidades de la administración pública federal y gobiernos estatales y municipales, no sujetos a reembolso. Se ocupan básicamente para sufragar gastos e inversiones en proyectos de alta rentabilidad social y baja o nula rentabilidad económica, y se agrupan en las siguientes opciones: subvenciones, aportaciones a gobiernos y dependencias, y estudios y asesorías.

La mayor parte de los recursos se destinó a la opción de aportaciones, con lo que prácticamente $80 \%$ fue para este fin. La distribución de los fondos por tipo de proyecto se muestra en la gráfica 4 .

2 Los fondos tienen un plazo generalmente de diez años, la mitad de inversión y el resto de desinversión, durante el cual de reintegran los rendimientos y las inversiones a los tenedores. 
Gráfica 4. Distribución de apoyos no recuperables

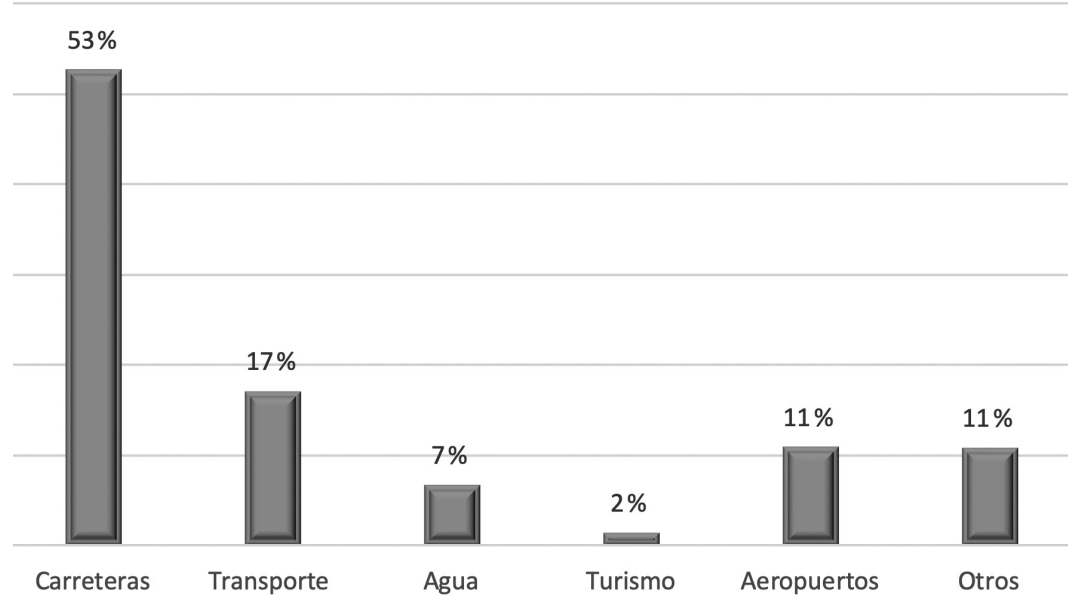

Fuente: Instituto Nacional de Transparencia, Acceso a la Información y Protección de Datos Personales con datos del Banco Nacional de Obras y Servicios Públicos.

De esta manera, los proyectos que han recibido mayor financiamiento corresponden al sector comunicaciones (carreteras y libramientos) y transporte (masivo), seguido por los correspondientes a aeropuertos.

Si bien la información descrita da una idea de cómo se han distribuido los recursos del Fonadin, es importante analizar cuál ha sido el resultado de la asignación de esos apoyos.

\section{Eficiencia en el uso de los recursos}

Como cualquier ejercicio de distribución de recursos públicos, los proyectos que ha financiado el Fonadin enfrentan diversas vicisitudes que, en muchos casos, terminan por diferir e inclusive no cumplir sus objetivos y metas.

Uno de los problemas de inicio es que las decisiones sobre el financiamiento de los proyectos se guían más por razones políticas, clientelares y de intereses particulares, que en respuesta a cuestiones técnicas y a los impactos económicos que deben y pueden generarse con este tipo de acciones de gobierno.

Otro aspecto relevante es que, si bien está claramente definido el marco regulatorio, en muchas obras los procesos de licitación se vuelven largos y costosos, desincentivando la participación de los potenciales oferentes. En 
este mismo sentido, conviene señalar que en diversas licitaciones los participantes, a efecto de ganar los proyectos, presentan posturas bajas con estudios de aforos e ingresos sobredimensionados, de manera que en el transcurso de las obras los presupuestos resultan insuficientes y se generan casos de sobrecostos y situaciones de ineficiencias en el mercado. Tal es la situación de un proyecto que tiene un apoyo del Fonadin y registra, tanto en sus aforos como en sus ingresos, prácticamente la mitad de lo que habían presupuestado en su plan de negocios.

El último elemento por considerar es la falta de planeación de largo plazo, que se origina tanto por los cambios institucionales de las administraciones federales y a nivel estatal y municipal, como por la falta de coordinación entre los tres niveles de gobierno. Como ejemplo, se tiene un proyecto que respalda el Fonadin en el estado de Chihuahua para la construcción e instalación de una planta de clasificación y separación de residuos, en donde participan varios municipios, y la conciliación de intereses y perspectivas no ha resultado del todo eficiente. Así, sin la planeación adecuada, se presenta una dispersión de las acciones públicas y la consecuente falta de integración de las políticas en su concepción, seguimiento y evaluación. De esta manera, al no contar con una estrategia intersectorial que tenga una visión transexenal, no se concretan los criterios de sostenibilidad y transversalidad en las políticas públicas de los servicios de infraestructura.

Si bien en cada etapa de desarrollo del proyecto pueden presentarse situaciones particulares, el resultado global ha sido una asignación ineficiente de los recursos públicos, que al ser limitados, generan altos costos de oportunidad en detrimento de otros proyectos. Con estas inequidades distributivas, la obra pública en infraestructura pierde su sentido como un instrumento al servicio del interés público y se convierte, en muchos casos, en una fuente de financiamiento de otros intereses.

Ante esta situación se identifica la necesidad de revisar las políticas y acciones que se están llevando a cabo en la materia, ya que la provisión insuficiente e ineficiente de capital para crear infraestructura se ha convertido en un hito a resolver en aras de sustentar la integración económica y social del país.

\section{Propuesta: hacia una nueva gobernanza del Fonadin}

Con base en los resultados del análisis de la situación de la infraestructura en México, se requiere un cambio en las pautas y un ajuste en los criterios para la aprobación de los proyectos que se apoyan con recursos del Fonadin. 
Para lograr este cambio, se hace preciso un cambio profundo de cómo se diseña, se financia, se implementa y se usa la infraestructura, lo que implica el cambio de la gobernanza misma del sector, es decir, en el conjunto de procesos tanto de toma de decisiones en el ámbito de la infraestructura como de la implementación de dichas decisiones, en los cuales actúan los mecanismos, procedimientos y reglas establecidas formal e informalmente por las instituciones (Cepal, 2016).

En este contexto se propone redefinir la gobernanza de Fonadin a partir de sus reglas de operación, para lo cual se precisa conceptualizar un esquema que le permita cumplir sus objetivos, ${ }^{3}$ a la vez que se oriente al uso eficiente de sus recursos. Para ello se proponen las siguientes directrices:

1. Enfocarse a desarrollar infraestructura en los sectores en los que la inversión equilibre el «encadenamiento» de valor social con el excedente marginal de la inversión. Ello requiere un análisis eficiente sobre los aspectos económicos y técnicos de los proyectos y, por lo tanto, de un menor peso de criterios y compromisos políticos en la selección de los mismos, lo que ayudaría a reducir los riesgos y la incertidumbre sobre su viabilidad operativa y financiera. La selección de los proyectos a financiar debe basarse en un esquema cuyos criterios de evaluación favorezcan una mayor conectividad entre las redes de infraestructura, reduzcan las asimetrías existentes entre sectores y regiones y generen espacios integrados que potencien la disponibilidad de factores y procesos productivos. A manera de propuesta se tiene el esquema sobre criterios de selección (figura 1):

3 Los objetivos de Fonadin, según se estable en sus reglas de operación, son los siguientes: maximizar y facilitar la movilización de capital privado a proyectos de infraestructura; Apoyar proyectos de gran impacto social y hacer bancables proyectos de alta rentabilidad social, pero poco atractivos para la iniciativa privada por su bajo retorno económico. 
Figura 1. Criterios de selección de proyectos

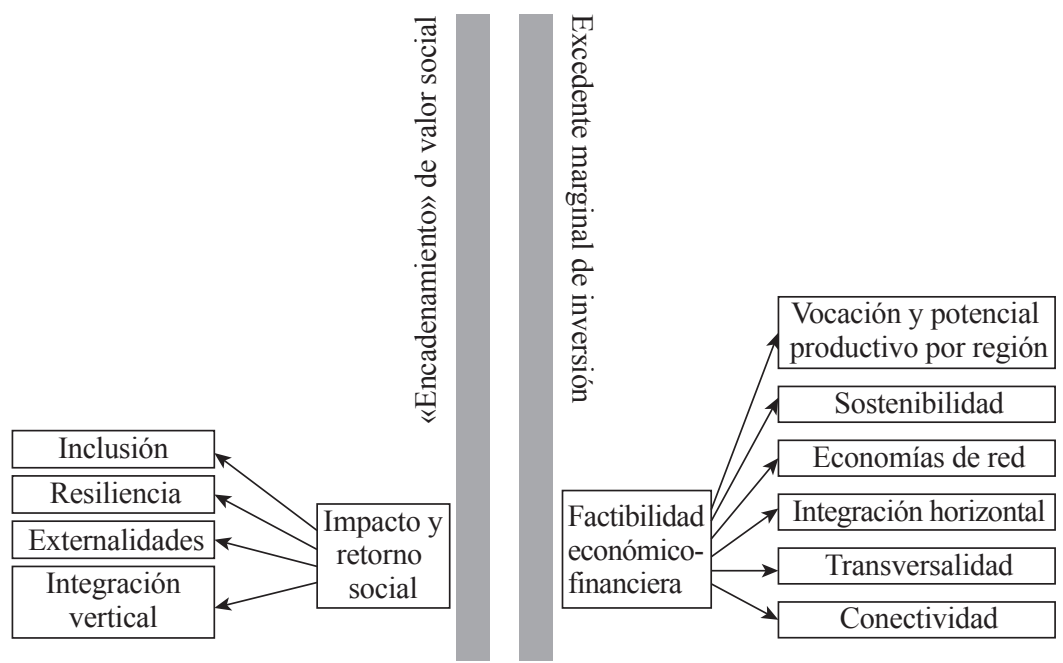

Fuente: elaboración propia.

En este proceso es necesario alinear la concepción, ejecución, seguimiento y evaluación de los proyectos, buscando la coherencia entre los objetivos y demandas de los grupos de interés públicos y privados, de modo que se asegure que los proyectos apoyados trasciendan los tiempos del calendario político de los tres órdenes de gobierno.

2. La evaluación de los proyectos a financiar debe tener como criterio rector la eficiencia y el costo de oportunidad del capital. Como ya se señaló, en el Fonadin hay un sesgo hacia los apoyos no recuperables, lo que ha generado situaciones de ineficiencia en el uso de los recursos; esto puede verificarse en el siguiente esquema (figura 2) para la selección y prelación de los proyectos, basado en el esquema de Hartley y Tisdell (1981), que tiene como referencia los conceptos de eficacia y eficiencia técnica y distributiva. ${ }^{4}$

4 Eficacia: grado de cumplimiento de las metas establecidas sin tener en cuenta los costos de los medios empleados para la consecución de los objetivos.

Eficiencia técnica: producir el máximo output con la menor cantidad de inputs como el trabajo, capital y tecnología.

Eficacia distributiva: es el output que maximiza el bienestar total, cuando en el equilibrio de mercado (precio igual a costo marginal) se maximiza el excedente del consumidor y del productor. 
Figura 2. Esquema de análisis y decisión

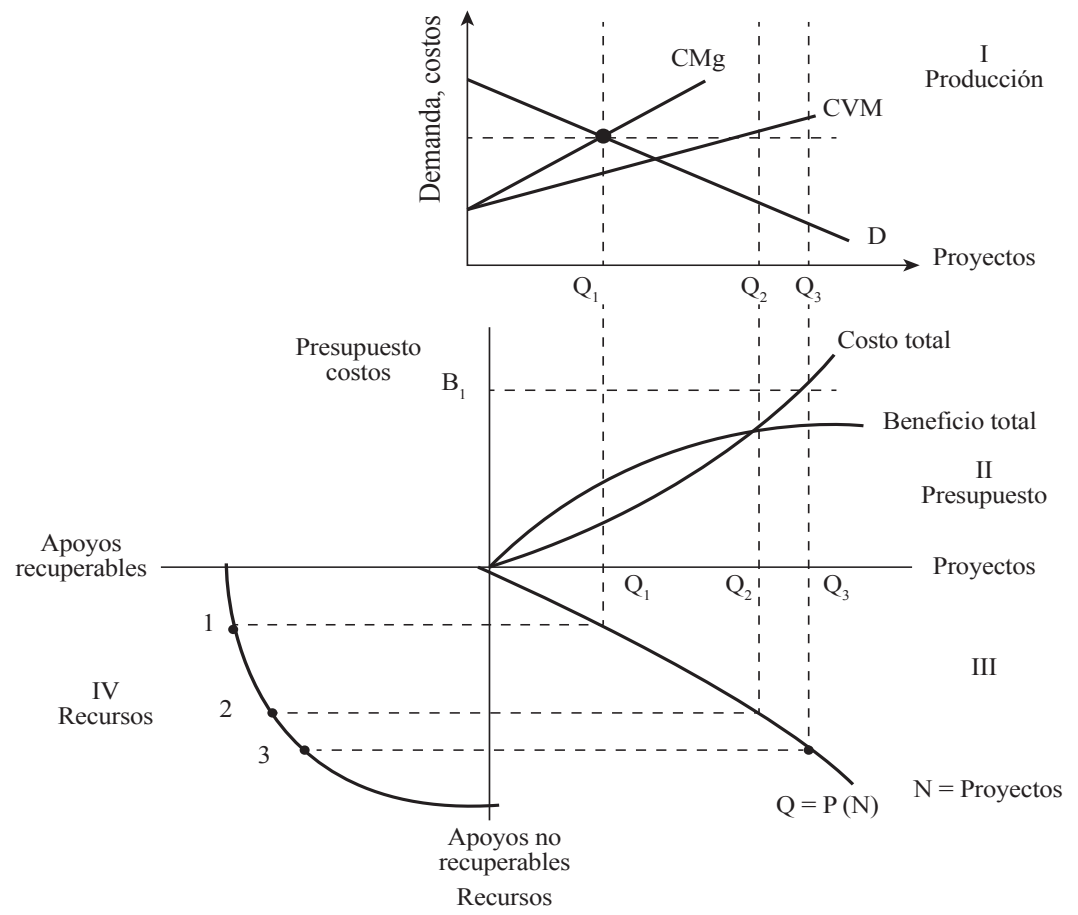

Fuente: elaboración propia con base en el esquema de Hartley y Tisdell (1981).

Como puede observarse, en la medida en que el Fonadin tiene restricción de recursos, necesariamente enfrenta costos de oportunidad en sus decisiones de financiamiento, lo cual se representa en el Cuadrante IV con la frontera eficiente de producción, que relaciona las alternativas de combinaciones de financiamiento de proyectos entre apoyos recuperables y apoyos no recuperables. Una vez seleccionada la combinación de ambas opciones, ello se refleja en la función de producción de proyectos del cuadrante III, cuyos costos y beneficios correspondientes se analizan en el cuadrante II. Finalmente, en la parte I del esquema se compara la elección de proyectos con los indicadores de la eficiencia distributiva del mercado.

Con la finalidad de analizar el funcionamiento del esquema, se tienen tres posibles combinaciones de proyectos (1, 2 y 3$)$. En el primer caso se trataría de una alternativa en que los recursos del Fonadin se destinan en mayor medida a proyectos del tipo de apoyos recuperables, es decir, aquellos que tienen una fuente para avalar su retorno. Por ello, al ubicarse en el punto donde la demanda es igual al costo marginal y el beneficio es mayor al costo, estaría asegurando una eficacia y una eficiencia distributiva y técnica. 
Por su parte, la combinación 2 tendría un equilibrio entre el costo y el beneficio totales, y en el punto 3 podría ubicarse la actual cartera de proyectos del Fonadin, que como ya se señaló, tiene una mayor participación de apoyos no recuperables (65\%), y con ello se tendría una ineficiencia distributiva toda vez que el costo medio resulta superior a la demanda.

A manera de conclusión, puede señalarse que el Fonadin debe reducir al máximo las subvenciones y destinar los apoyos no recuperables a proyectos con impacto regional y que generen sinergias entre las redes ya instaladas, con lo que se originarán externalidades de red y de alcance. Lo importante es evitar la multiplicidad de infraestructuras y favorecer una mayor conectividad, reduciendo así las asimetrías entre las distintas regiones del país.

El lineamiento propuesto está incluido en las Reglas 8 y 9 de las ROP, pero también es necesario considerar el impacto regional como criterio de selección de proyectos. Con esta propuesta se esperaría un movimiento del punto 3 al punto 2 en el esquema.

3. Para cumplir con el objetivo de facilitar la movilización de capital privado a proyectos de infraestructura, en principio es necesario identificar la infraestructura como una clase especial de activo ya que, a diferencia de otros activos fijos, tiene un elevado riesgo inicial debido a costos de construcción no previstos, incertidumbre sobre el nivel estimado de la demanda que impacta los niveles proyectados de ingresos y costos, además de la imposibilidad de reconvertir este tipo de activos a usos alternativos.

Por ello es importante fomentar la participación de la banca comercial y los inversionistas institucionales en este proceso, canalizando el ahorro nacional hacia este sector. De esta manera, el Fonadin debe destinar mayores recursos a los fondos de inversión y a las fibras, porque son una excelente alternativa para financiar proyectos de infraestructura con alto impacto social y tienen además un menor riesgo sobre su viabilidad económica, ya que está implícito el criterio de rentabilidad financiera en su selección. Actualmente, estos instrumentos de inversión representan el $14 \%$ del total de la cartera total del Fonadin y, de hecho, ofrecen a los tenedores tasas internas de retorno en promedio superiores a $15 \%$, con lo que se generan recursos para otros financiamientos, reduciendo el costo de oportunidad del capital.

A manera de ejemplo para reforzar lo anterior, hay un par de proyectos en el sector agua en donde el Fonadin aportó una subvención y, además, en la estructura de capital ${ }^{5}$ de los proyectos participa un fondo de inversión en

5 La estructura de capital de los proyectos está integrada por: apoyo Fonadin, capital de riesgo, créditos y aportación estatal y municipal. 
el que el mismo Fonadin tienen recursos invertidos, lo cual resulta ineficiente, dado que podrían distribuirse estos recursos a otras alternativas.

Otro aspecto relevante es que con esta opción, al no haber una relación directa del Fonadin con los beneficiarios, es posible aplicar las cláusulas de penalización establecidas en los instrumentos jurídicos que sustentan los apoyos, lo que no siempre es factible en el caso de los apoyos no recuperables por cuestiones políticas.

Para este tercer lineamiento, el sustento está en las Reglas 10,11 y 26 de las ROP y con su aplicación se esperaría un movimiento del punto 3 al 1 en la figura 2 .

\section{Conclusiones}

Del análisis efectuado en este artículo, es posible señalar que al contar con redes de infraestructura eficientes se asegura el acceso, que constituye el elemento central de la integración del sistema económico, social y territorial en el espacio geográfico de un país.

México no es la excepción y se han destinado importantes recursos para este fin, pero no han sido suficientes para sustentar el desarrollo y crecimiento económicos. De hecho, en la clausura de la 82 Convención Bancaria, el secretario de Hacienda y Crédito Público reconoció que la inversión pública es menor a $3 \%$ en comparación con el PIB y que en esa medida «Una economía en donde la inversión en infraestructura pública tiene tan bajos porcentajes, no tiene en el largo plazo ninguna viabilidad para poder alcanzar un crecimiento robusto» (Mendoza, 2019).

En este contexto, el Fonadin ha desempeñado un papel relevante, ya que es uno de los brazos ejecutores del gobierno en el desarrollo de infraestructura, pero ante los resultados obtenidos, se propone cambiar la perspectiva a través de un proceso de nueva gobernanza, a efecto de que la selección de proyectos se haga sobre otras bases y criterios, de manera que trasciendan los tiempos políticos, se potencien las interrelaciones geográficas y se maximicen los efectos transversales en distintos sectores de la economía. Con ello se tendrán elementos y argumentos tanto para evaluar el diseño y la puesta en marcha de las políticas públicas, como para resolver las controversias que se presentan en esta materia, como es el caso de la construcción del Nuevo Aeropuerto Internacional en Texcoco.

Asimismo, parece ineludible la búsqueda de fórmulas de financiamiento para el desarrollo de la infraestructura y, en este sentido, es importante incentivar la participación de capital privado para estos proyectos, tomando en cuenta que los fondos de inversión y las fibras han demostrado ser una buena alternativa. 


\section{El autor}

Daniel Ordóñez Bustos es doctor en Economía, con experiencia profesional en el campo de las telecomunicaciones y en la evaluación y financiamiento de proyectos. En materia de investigación ha escrito artículos y varios textos, uno de los cuales resultó ganador de la $1^{\text {a }}$ Convocatoria Nacional NYCE. En el ámbito académico fue profesor del Departamento de Economía de la Universidad Iberoamericana durante catorce años y actualmente participa en el Programa de Maestría en Línea de la Universidad Anáhuac.

Correo electrónico: daordone@gmail.com 


\section{Bibliografia}

Andrade Hernández, J.M. y Lugo Delgadillo, M. (2018). «Qué tan factible es aumentar el acervo de infraestructura en México: una propuesta de inversión de largo plazo». Instituto Belisario Domínguez. Recuperado de http://bibliodigitalibd.senado.gob.mx/handle/123456789/3969

Arrow, K. J. y Kurz, M. (1970). «Public investment, the rate of return and optimal fiscal policy». Baltimore: Johns Hopkins University Press.

Aschauer, D.A. (1989). «Is public expenditure productive?». Journal of Monetary Economics 23: 177-200.

Banco Nacional de Obras y Servicios Públicos, Banobras (2018). Informe anual 2017. México. Recuperado de: https://www.gob.mx/cms/uploads/ attachment/file/416642/Informe_Anual_Consolidado.pdf

Calderón, C. y Servén, L. (2002), «The output cost of Latin America's infrastructure gap»; Central Bank of Chile, Working Paper (186). Recuperado de: https://webimages.iadb.org/publications/spanish/document/ Financiamiento-de-la-infraestructura-en-Am\%C3\%A9rica-Latina-y-elCaribe- $\% \mathrm{C} 2 \% \mathrm{BFC} \% \mathrm{C} 3 \% \mathrm{~B} 3 \mathrm{mo}-\mathrm{cu} \% \mathrm{C} 3 \% \mathrm{~A} 1$ nto-y-qui\%C3\%A9n.pdf

Centro de Estudios Económicos del Sector de la Construcción (2017). «Ranking mundial de competitividad en infraestructura 2017-2018 (World Economic Forum)» (documento en línea). Recuperado de: https://www. cmic.org. $\mathrm{mx} / \mathrm{cmic} / \mathrm{ceesco} / 2018 / \mathrm{RANKING} \% 20 \mathrm{MUNDIAL} \% 20 \mathrm{DE} \% 20$ COMPETITIVIDAD\%204.0\%20EN\%20INFRAESTRUCTURA\% 202018-2019.pdf

Comisión Económica para América Latina y el Caribe, CEPAL (2011). «Caracterización de la brecha de infraestructura económica en América Latina y el Caribe». Boletín FAL (293). Recuperado el 25 de febrero de 2019 de: https://repositorio.cepal.org/bitstream/handle/11362/36132/1/ FAL-293-WEB_es.pdf

Comisión Económica para América Latina y el Caribe, CEPAL (2016) «La gobernanza de la infraestructura a favor del desarrollo basado en la igualdad y la sostenibilidad» (sitio de internet). Recuperado el 12 de febrero de 2019 de: https://www.cepal.org/es/temas/infraestructura/lagobernanza-la-infraestructura-favor-desarrollo-basado-la-igualdadla-sostenibilidad

Comisión Económica para América Latina y el Caribe, CEPAL (15 de mayo de 2017). «Inversión en infraestructura en países de América Latina y el Caribe se mantiene por debajo de necesidades de la región» (sitio de internet). Recuperado el 12 de febrero de 2019 de: https://www.cepal. org/es/noticias/inversion-infraestructura-paises-america-latina-caribese-mantiene-debajo-necesidades-la

Fondo Nacional de Infraestructura, Fonadin (s/f) Reglas de operación. Fideicomiso núm. 1936. Fondo Nacional de Infraestructura. Recuperado de: 
http://www.fonadin.gob.mx/wp-content/uploads/2016/08/Reglas_de Operacion_2015_11.pdf

Hartley, Keith y Tisdell, Clem (1981). «Micro-Economic Policy». Nueva York: John Wiley \& Sons.

Mendoza Escamilla, Verónica (22 de marzo de 2019). «Sin inversión en infraestructura, no es viable crecer más: Urzúa». Forbes. Recuperado el 25 de marzo de 2019 de https://www.forbes.com.mx/sin-inversion-eninfraestructura-no-es-viable-crecer-mas-urzua/

Niskanen, W. (1971). «Bureaucracy and Representative Government». Chicago: Aldine Atherton.

Noriega, A. y Fontenla, M. (2007). «La infraestructura y el crecimiento económico en México». El Trimestre Económico . 74 (296). Recuperado de: http://www.eltrimestreeconomico.com.mx/index.php/te/article/ view/386/583

Organización para la Cooperación y el Desarrollo Económicos, OCDE (2016). «Hacia infraestructuras exitosas. Diez retos clave para la gobernanza y opciones de política». Recuperado de: https://www.oecd.org/ gov/budgeting/hacia-infraestructuras-exitosas.pdf

Peiro Ucha, Alfonso (s/f) «Deuda senior». Economipedia (sitio de internet). Recuperado el 15 de febrero de 2019 de: https://economipedia.com/ definiciones/deuda-senior.html

Pindick, R. y Rubinfeld, D. (1995). «Microeconomía». España. Prentice Hall. Secretaría de Hacienda y Crédito Público, SHCP (2016). «Proyectos APP». Recuperado el 5 de marzo de 2019 de: https:/www.gob.mx/shcp/accionesy-programas/proyectos-app

Serebrisky, T., Suárez-Alemán, A. y Ramírez. M. (2015) «Financiamiento de la infraestructura en América Latina y el Caribe: ¿Cómo, cuánto y quién?». Banco Interamericano de Desarrollo. Recuperado de: https:// webimages.iadb.org/publications/spanish/document/Financiamientode-la-infraestructura-en-Am\%C3\%A 9rica-Latina-y-el-Caribe$\% \mathrm{C} 2 \% \mathrm{BFC} \% \mathrm{C} 3 \% \mathrm{~B} 3 \mathrm{mo}-\mathrm{cu} \% \mathrm{C} 3 \% \mathrm{~A} 1$ nto-y-qui $\% \mathrm{C} 3 \% \mathrm{~A} 9 \mathrm{n}$.pdf

Standard \& Poor's (2015). «Global Infrastructure Investment: Timing Is Everything (and Now is the Time)». Standard \& Poor's Ratings Services. Recuperado de: https://pdfsecret.com/download/global-infrastructureinvestment-trade-and-forfaiting-review_59fc33b1d64ab28ae265cb1f_pdf

Tourliere, M. (2019). «El Fonadin, otra descomunal veta de corrupción del gobierno de Peña Nieto». Proceso. (2203): 36-38. 